\title{
Prevalence of avian influenza, Newcastle disease, and infectious bronchitis viruses in broiler flocks infected with multifactorial respiratory diseases in Iran, 2015-2016
}

\author{
Habibbolah Haji-Abdolvahab ${ }^{1} \cdot$ Arah Ghalyanchilangeroudi $^{2} \cdot$ Alireza Bahonar $^{1}$ (D) - Seyed Ali Ghafouri ${ }^{3}$. \\ Mehdi Vasfi Marandi ${ }^{4} \cdot$ Mohammad Hosein Fallah Mehrabadi $^{5} \cdot$ Farshad Tehrani $^{3}$
}

Received: 9 August 2018 / Accepted: 18 October 2018 / Published online: 30 October 2018

(C) Springer Nature B.V. 2018

\begin{abstract}
In this study, the prevalence and spatial distribution of Newcastle disease, infectious bronchitis, and avian influenza have been evaluated in commercial broiler farms in 31 provinces in Iran. In this survey, a total of 233 affected broiler chicken farms were sampled. The infectious bronchitis virus (alone) was detected with highest frequency in $60 \mathrm{farms}$, and separately or combined with other agents, in 110 farms; Newcastle disease virus, separately, was detected in 28 farms, and in 63 farms separately or combined with other infectious agents; and avian influenza H9N2 was detected in 22 farms separately and in 51 farms separately or concomitant with other infectious agents. The sample tested negative for all $\mathrm{H} 5$ serotypes. The results of the present study show that the most prevalent avian viral infectious disease contributing to respiratory syndromes in broiler farms in Iran was infectious bronchitis due to infectious bronchitis virus serotypes variant 2 and 793/B. On the other hand, combined with the alternation of dominant viruses and circulating strains, flocks are exposed to unremitting anamorphic viral infections. Thus, the permanent monitoring of cases that have occurred and the review of vaccination plans of affected flocks every year are some of the necessary measures needed for strategic control of respiratory syndrome in broilers. It is noteworthy that execution of epidemiologic examinations on the cogent factors of prevalence of this syndrome and defeat of vaccination strategy in the flocks is urgent and has to be fulfilled on the definite causes of time.
\end{abstract}

Keywords Avian influenza $\cdot$ Infectious bronchitis $\cdot$ Newcastle disease $\cdot$ Respiratory syndrome

\section{Introduction}

Over the past years, the Iranian poultry industry, and specially the broiler breeding, has grown significantly, in the way that

Alireza Bahonar

abahonar@ut.ac.ir

1 Department of Food Hygiene \& Quality Control, Faculty of Veterinary Medicine, University of Tehran, Tehran, Iran

2 Department of Microbiology and Immunology, Faculty of Veterinary Medicine, University of Tehran, Tehran, Iran

3 Department of Health and Management of Poultry Diseases, Iran Veterinary Organization, Tehran, Iran

4 Department of Poultry Diseases, Faculty of Veterinary Medicine, University of Tehran, Tehran, Iran

5 Department of Poultry Diseases, Razi Vaccine and Serum Research Institute, Agricultural Research, Education and Extension Organization (AREEO), Tehran, Iran the production of poultry meat has risen from 110,000 tons in 1974 to 210,000 tons in 2016. The development of this industry has increased the density (concentration) of poultry farms in some parts of the country. Poultry farming ranging from extensive free range to intensive integrated production systems has provided conditions for the incidence of complex multifactorial infections such as bacterial, mycoplasma, and viral ones. In many cases, respiratory problems are due to infection with several infectious factors rather than with single one (Swayne et al. 2013). Avian influenza (AI), Newcastle disease (ND), and infectious bronchitis (IB) are the major viral infections in broilers that present with respiratory signs.

In Iran, respiratory diseases in poultry due to AIV, NDV, and IBV cause constant mortality in broilers especially. These infections occur either in the solitary form or in combination with multifactorial infections. Evidence indicates that most of these factors are synergistic and potentate themselves in infected flocks. In recent years, despite national and domestic prevention and control measures against AI, ND, and IB, 
coupled with widespread usage of diverse vaccines, outbreaks of these viral diseases still cause respiratory syndromes in broiler flocks resulting in high mortalities annually. Based on available statistics from the Iran Veterinary Organization (IVO), maximum economic damage in broiler flocks is related to losses arising from respiratory complications (Ebadzadeh et al. 2015).

Avian influenza caused by strain H9N2 is endemic in many Asian and the Middle East countries including Iran (Naeem et al. 2007; Nili and Asasi 2003). The causal agent is avian influenza virus type A that belongs to the Orthomyxoviridae family. Newcastle disease caused by avian paramyxovirus I (APMV-I) is also endemic in Asian and the Middle East countries. Co-infections in broiler flocks with AIV and NDV result in great economic losses due to severe morbidity and mortality rates. A third co-infection with IBV worsens the scenario. The causal agent of this disease belongs to the coronavirus subtype and the gamma coronavirus genus (OIE 2017).

In this study, the evaluation of prevalence and spatial distribution of Newcastle disease, infectious bronchitis, and avian influenza H9N2 subtype has been carried out for commercial broiler farms in Iran.

\section{Materials and methods}

\section{Study population and design}

The industrial broiler farms are present in 31 Iranian provinces. Based on the Geographical Information System database of poultry diseases of IVO (Iran Vet GIS), there are nearly 25,000 broiler farms in the country, of which (according to the Ministry of Agriculture statistics) 19,548 have formal operating certificates (Ebadzadeh, Ahmadi, Mohammadnia Afruzi, Taghani, Moradi Eslami, and Yari 2015). Based on Iran Vet GIS, on an annual average, nearly 19,000 farms of the total 25,000 broiler farms are active with about four breeding periods in a year. The aggregation of broiler farms in the various provinces of Iran shows that the highest number is located in Mazandaran and Esfahan provinces. In addition, the number of breeding periods in these farms during each year is distinct on disparate zones (different areas of the country), so that it varies from three times in most central provinces with warm and dry climate up to five times in the northern provinces with moderate and wet climate, on average per year. The study population included all active (in production) broiler farms (based on Iran Vet GIS) that were infected with respiratory diseases as a new case during the study period. A farm infected with respiratory disease was defined as a flock with core clinical signs such as depression, snickering, sneezing, coughing, dyspnea, rattling, and nasal discharges with or without diarrhea or central nervous system signs (wing paralysis, legs paralysis, or twisted neck) that entailed rising mortality rates for at least 3 days.

The sampling period in this cross-sectional study was from 21st of April 2015 to 19th of March 2016. All broiler farms in Iran are under the supervision of a private Vet as a farm Vet or clinician, in groups of one to several, based on farm population and location. The farmers and their Vet supervisors must give online prompt report (registered in GIS) when a farm is involved in serious problems, including important diseases as defined by IVO as a National Assignment. So the study population including infected farms was self-reported by farmers or their private Vets supervisor, in addition to farms that were reported following the active surveillance programs of IVO by state Vets.

\section{Sample size and data collection}

According to data registered with the Iranian Vet GIS, the prevalence of IB, ND, and $\mathrm{AI}$ in the previous year in our study was $18.7 \%, 9.6 \%$, and $8.2 \%$, respectively. Thus, the prevalence of IB (18.7\%) was chosen to calculate the total sample size, with 5\% accuracy and 95\% confidence level.

In this survey, the sampling method was proportional random sampling based on the proportion of each province broiler farms that were in production. The number of required samples for each province was assigned according to the proportion of its active (in production) broiler farms present for at least past 3 years, according to Iranian Vet GIS. So all the infected farms were listed by the province and then samples were selected using the simple random selection from each province list. Based on the calculated sample size of 233 , a total population of 76,000 and size of $x$ (proportion of each province) and using the formula, the number of farms to be sampled per province is calculated as follows:

Number of farms to be sampled in each province

$$
=\left(\frac{x}{N}\right) \times n \text {. }
$$

In each affected farm, 7-10 recently dead birds or birds with clinical symptoms were sampled. The collected samples consisted of trachea tissue, lungs, brain, spleen, cecal tonsil, and kidneys. The samples were delivered to the IVO central laboratory. After recording the complete characteristics, the samples were preserved and stored at $\mathrm{a}-70{ }^{\circ} \mathrm{C}$ freezer for further examinations.

After completion of the tests, the related results were received from the IVO central laboratory, along with the data of total sample mortalities at the end of that particular breeding season. 


\section{Laboratory test processes}

In this study, the samples of the trachea and lung; the samples of the spleen, cecal tonsil, and kidney; and brain samples from each farm were segregated, after which $10 \%$ PBS suspensions were prepared. After centrifugation at a low rate, $1 \mathrm{ml}$ of supernatant was collected and used for tests. The tests were conducted parallelly and all samples from each farm were tested. We considered a test positive if the result of each sample was positive regardless of other sample results. For cases with clinical nervous signs, the brain samples underwent additional testing.

\section{RNA extraction}

RNA extraction was done on the prepared suspensions using a High Pure Viral Nucleic Acid Kit (Roche, Germany) according to the manufacturer's protocol. The extracted RNA was divided into three equivalent parts and each part was used for $\mathrm{AI}, \mathrm{IB}$, and $\mathrm{ND}$ virus diagnostic tests.

For diagnosis of avian influenza, infectious bronchitis, and Newcastle disease, molecular testing was done according to recommended methods.

For diagnosis of H9N2 and H5 avian influenza, we used the method of real-time RT-PCR (Monne et al. 2008).

For ND detection, the RT-PCR method using two pairs of primers was performed as described by Kant et al.; $A B$ primers were used to confirm the existence of the ND virus. To confirm ND velogenic viruses, the positive samples were then tested by RT-PCR using AD primers. If a sample in the first RT-PCR was positive but negative in the second, the detected virus was classified as a velogenic (Kant et al. 1997).

For IBV detection, real-time PCR was done based on 5' UTR. We used the amplification kit (Bioneer, South Korea), with the forward primer 5'GCTTTTGAGCCTAGCGTT3', reverse primer 5'GCCATGTTGTCACTGTCTATTG3', and Taqman ${ }^{\circledR}$ dual-labeled probe FAMCACCACCAGAACCT GTCACCTC-BHQ1 (Callison et al. 2006). For IBV genotyping, a nested PCR assay was done and then sequencing was performed with the primers (both directions) as used in the PCR (Bioneer Co., Korea). We did nucleotide BLAST (NCBI) the results of edited obtained sequences for detection of IBV genotypes.

\section{Results}

A total of 233 infected broiler farms were sampled in the study. All farms were vaccinated for ND and IB but for AIH9N2, some farms were vaccinated. The sizes of farms were different from 5000 to $1,000,000$ birds. The breeding system of the farms was different too based on the region and the farm building date.
The most sampled farms were from Mazandaran, Esfahan, and Khorasan Razavi with 19, 22, and 23 farms, respectively. The provinces of Tehran, North Khorasan, SistanBaluchestan, and Southern Kerman with only one farm each were the lowest sampled. The distribution of sampled farms is shown in Table 1 province-wise.

The mean disease start age for broiler farms infected to IB was 23 rd day (95\% CI 21-25), for ND was 26th day (95\% CI 23-29), and for H9N2 was 25th day (95\% CI 22-29).

Alone, the infectious bronchitis virus was detected of highest concentration in 60 farms $(25.7 \%)$ and alone or together with the other agents, in 110 farms $(49.3 \%)$. A total of 85 farms (36.5\%) were positive for Newcastle viruses with 63 velogenic cases; as lone virus, it was detected in 28 farms (12\%), and in 63 farms (27\%) alone or together with the other infectious agents; and avian influenza H9N2 was detected in 22 farms (9.4\%) alone and in 51 farms (21.9\%) alone or concomitant with the other infectious agents (Table 1). All samples were tested negative for $\mathrm{H} 5$ subtype viruses (Fig. 1).

Out of 60 isolated infectious bronchitis viruses, 19 viruses (31.6\%) were 793/B genotype, 17 viruses (28.3\%) were variant 2 genotype (IS/1494), 10 viruses $(16.6 \%)$ were Massachusetts, five viruses (8.3\%) were QX genotype, three viruses (5\%) were IR genotype, three viruses (5\%) IR2 genotype, and another three viruses $(5 \%)$ were of mix variants.

The highest mean mortality rate was $61.7 \%$ as observed in the farms infected with H9N2 AI, NDV, and IBV, simultaneously; the lowest mean mortality rate with $31.14 \%$ was observed in the farms affected with H9N2 AI (Chart 1). The highest percentage of mortality was in Sistan-Baluchestan $(82 \%)$ and Kohgiluyeh-Boyerahmad provinces $(81.51 \%)$, and the lowest percentages were in Lorestan (13.20\%), Bushehr (12.5\%), and Northern Khorasan (4.4\%).

\section{Discussion}

Over the past years, respiratory diseases have been one of the most critical problems for national commercial broiler farms. Of these, infectious bronchitis, Newcastle disease, and H9N2 influenza have been the major endemic diseases in Iran with numerous studies carried out in this regard (Hadipour and Golchin 2011; Hosseini et al. 2015; Hosseini et al. 2014; Pourbakhsh et al. 2008; Seifi et al. 2010). Furthermore, these diseases were found to be some of the most critical factors for infections in the poultry industry, particularly in broilers, in other countries too (Hassan et al. 2016; Naeem et al. 2007; Xia et al. 2017).

This study showed infectious bronchitis to be the most significant agent of frequent respiratory syndrome of the studied farms in spite of widespread vaccination drives at these sites. Previous studies have recognized the contrasting 
Table 1 The frequency of avian Influenza H9N2, Infectious bronchitis and Newcastle Disease in broiler farms infected to respiratory complex in Iran, 2015-2016

\begin{tabular}{|c|c|c|c|c|c|c|c|c|c|c|c|c|}
\hline \multirow[t]{2}{*}{ Province } & \multirow[t]{2}{*}{ Sampled farms } & \multicolumn{7}{|c|}{ Single and mixed viral infection } & \multirow[t]{2}{*}{ None } & \multicolumn{3}{|c|}{ Total $(\%)$} \\
\hline & & $\begin{array}{l}\text { AI- } \\
\text { H9 }\end{array}$ & IB & ND & IB + AI-H9 & $\mathrm{IB}+\mathrm{ND}$ & $\mathrm{ND}+\mathrm{AI}-\mathrm{H} 9$ & $\mathrm{IB}+\mathrm{ND}+\mathrm{AI}-\mathrm{H} 9$ & & ND & IB & $\begin{array}{l}\text { AI- } \\
\text { H9N2 }\end{array}$ \\
\hline Mazandaran & 23 & 2 & 7 & 2 & 2 & 3 & 1 & 1 & 5 & 30.4 & 56.5 & 26.1 \\
\hline Esfahan & 22 & 6 & 0 & 4 & 2 & 1 & 0 & 0 & 9 & 22.7 & 13.6 & 36.4 \\
\hline Razavi Khorasan & 19 & 3 & 4 & 3 & 1 & 6 & 1 & 0 & 1 & 52.6 & 57.9 & 26.3 \\
\hline Fars & 17 & 3 & 6 & 0 & 2 & 1 & 1 & 0 & 4 & 11.8 & 52.9 & 35.3 \\
\hline Yazd & 13 & 1 & 5 & 0 & 0 & 2 & 1 & 0 & 4 & 23.1 & 53.9 & 15.4 \\
\hline East-Azarbayegan & 11 & 0 & 3 & 2 & 2 & 0 & 0 & 0 & 4 & 18.2 & 45.5 & 18.2 \\
\hline Qom & 11 & 0 & 5 & 1 & 2 & 0 & 0 & 1 & 2 & 18.2 & 72.7 & 27.3 \\
\hline Hamedan & 10 & 1 & 2 & 1 & 0 & 0 & 0 & 0 & 6 & 10.0 & 20.0 & 10.0 \\
\hline Khuzestan & 9 & 0 & 2 & 2 & 0 & 1 & 0 & 0 & 4 & 33.3 & 33.3 & 0.0 \\
\hline West-Azarbayegan & 8 & 1 & 0 & 0 & 0 & 4 & 0 & 0 & 3 & 50.0 & 50.0 & 12.5 \\
\hline Kordestan & 8 & 1 & 4 & 0 & 0 & 1 & 0 & 0 & 2 & 12.5 & 62.5 & 12.5 \\
\hline South Khorasan & 7 & 0 & 3 & 2 & 0 & 0 & 1 & 0 & 1 & 28.6 & 100.0 & 28.6 \\
\hline Qazvin & 7 & 0 & 1 & 1 & 3 & 0 & 0 & 0 & 2 & 28.6 & 42.9 & 28.6 \\
\hline Kerman & 7 & 0 & 1 & 1 & 1 & 0 & 0 & 1 & 3 & 14.3 & 14.3 & 28.6 \\
\hline Lorestan & 7 & 2 & 1 & 1 & 0 & 0 & 0 & 0 & 3 & 14.3 & 57.1 & 42.9 \\
\hline Markazi & 7 & 0 & 3 & 0 & 2 & 2 & 0 & 0 & 0 & 42.9 & 42.9 & 14.3 \\
\hline Ilam & 5 & 2 & 1 & 1 & 1 & 0 & 0 & 0 & 0 & 80.0 & 20.0 & 0.0 \\
\hline Kermanshah & 5 & 0 & 3 & 0 & 0 & 0 & 0 & 0 & 2 & 0.0 & 60.0 & 0.0 \\
\hline Gilan & 5 & 0 & 0 & 3 & 0 & 1 & 0 & 0 & 1 & 20.0 & 40.0 & 60.0 \\
\hline Zanjan & 4 & 0 & 0 & 2 & 1 & 0 & 0 & 0 & 1 & 50.0 & 25.0 & 25.0 \\
\hline Semnan & 4 & 0 & 1 & 1 & 0 & 0 & 0 & 0 & 2 & 25.0 & 25.0 & 0.0 \\
\hline Golestan & 4 & 0 & 0 & 0 & 1 & 1 & 0 & 0 & 2 & 25.0 & 75.0 & 0.0 \\
\hline Hormozgan & 4 & 0 & 2 & 0 & 0 & 1 & 0 & 0 & 1 & 25.0 & 50.0 & 25.0 \\
\hline Ardabil & 3 & 0 & 2 & 0 & 0 & 0 & 0 & 0 & 1 & 66.7 & 33.3 & 0.0 \\
\hline Chaharmahal & 3 & 0 & 0 & 1 & 0 & 1 & 0 & 0 & 1 & 0.0 & 66.7 & 0.0 \\
\hline Alborz & 2 & 0 & 0 & 0 & 0 & 0 & 0 & 1 & 1 & 50.0 & 100.0 & 0.0 \\
\hline Booshehr & 2 & 0 & 1 & 0 & 0 & 0 & 0 & 0 & 1 & 0.0 & 50.0 & 0.0 \\
\hline Kohgilooyeh & 2 & 0 & 1 & 0 & 0 & 1 & 0 & 0 & 0 & 50.0 & 50.0 & 50.0 \\
\hline Tehran & 1 & 0 & 1 & 0 & 0 & 0 & 0 & 0 & 0 & 0.0 & 0.0 & 0.0 \\
\hline South Kerman & 1 & 0 & 1 & 0 & 0 & 0 & 0 & 0 & 0 & 0.0 & 100.0 & 0.0 \\
\hline North Khorasan & 1 & 0 & 0 & 0 & 0 & 0 & 0 & 0 & 1 & 0.0 & 100.0 & 0.0 \\
\hline Sistan & 1 & 0 & 0 & 0 & 0 & 0 & 0 & 0 & 1 & 0.0 & 0.0 & 0.0 \\
\hline Total & 233 & 22 & 60 & 28 & 20 & 26 & 5 & 4 & 68 & 27.0 & 47.2 & 21.9 \\
\hline
\end{tabular}

serotypes of bronchitis in the country. In Iran, Mass serotype plus genotype 793/B have been reported (Jackwood 2012).

In the present investigation as well as in several other studies in Iran, 793/B was detected as the dominant serotypes and the principal virus of circulation. Pourbakhsh et al. conducted a study in 150 farms, wherein infectious bronchitis was found to be the dominant virus in $73 \%$ cases. In their study, the genotype 793/B was detected in $83 \%$ of farms, which were IB positive. In addition, another identified genotype was Massachusetts (Pourbakhsh et al. 2008). In a study in 2010 in Iran, $40 \%$ of sampled farms (12 farms out of 30 ) were infected with infectious bronchitis, 11 samples were genotype 4/91, and only one case was Massachusetts (Seifi et al. 2010).

In the investigation of Hosseini et al. carried out over the period 1994-2004, the serotype 793/B was tracked as the dominant serotype in the country (Hosseini et al. 2015).

In study that was done by Modiri et al., bronchitis has posed to be a challenge in broiler farms of Iran's neighboring countries as well (Modiri Hamadan et al. 2017). In an experiment relevant to Iraq during the years 2013-2014, 32 samples of IB virus were identified from 100 samples collected. This survey also identified variant 2 as the dominant virus (Seger 
Fig. 1 Distribution of broiler sampled farms for respiratory disease in Iran, 2015-2016

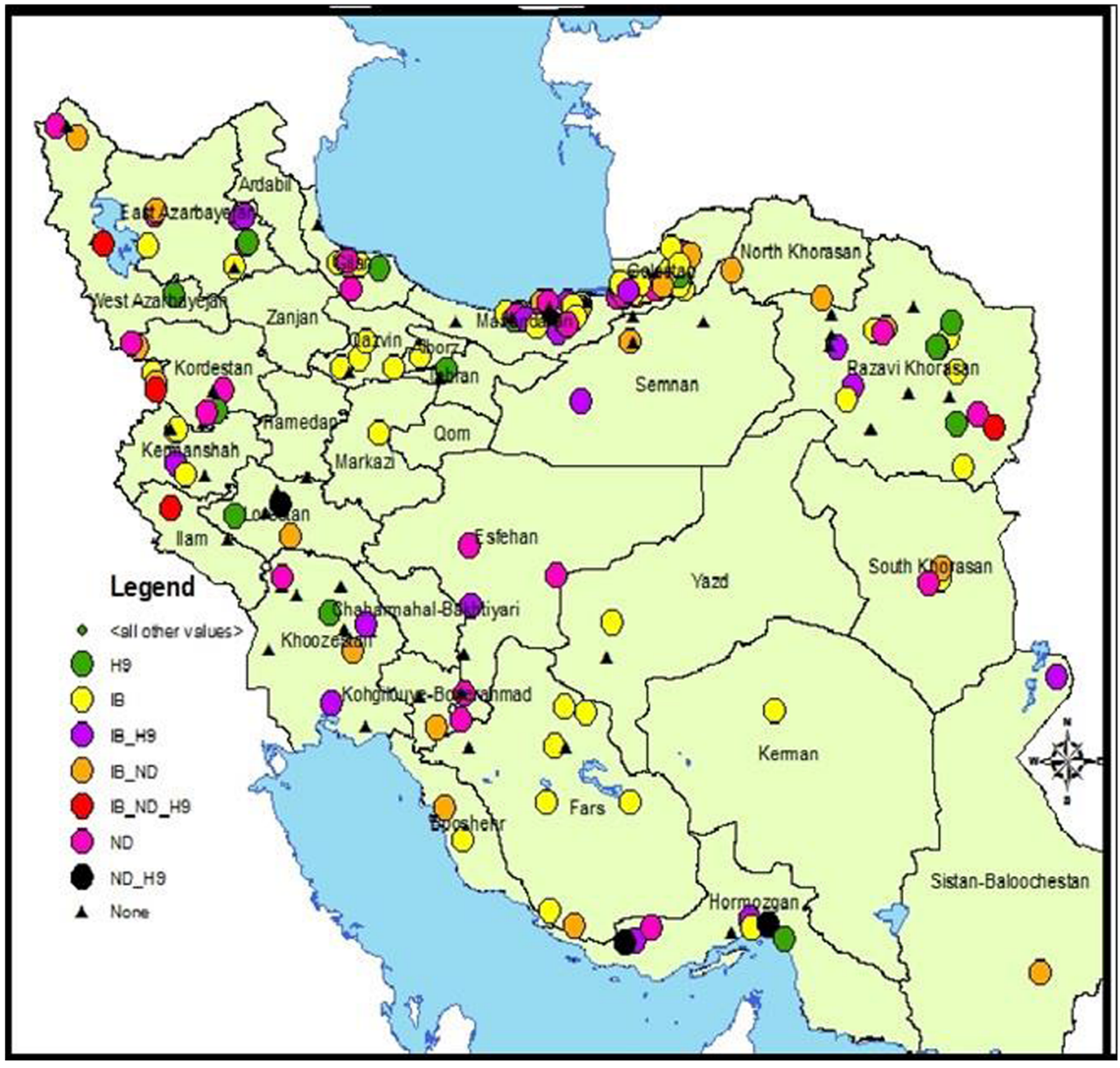

et al. 2016). In Egypt (Hassan et al. 2016), infectious bronchitis alone and combined with other factors infected with respiratory diseases in $18.6 \%$ and $86 \%$, respectively, has been pinpointed in 86 broiler chicken farms. Furthermore, the concomitant infection of bronchitis with avian influenza H9N2 was found to be the most common infection with $41.9 \%$ (Hassan et al. 2016).

After conclusive testing in Iran, the flocks of infected birds have been vaccinated against infectious bronchitis (Aghakhan et al. 1994). However, the main challenge in control of IB is
Chart 1 Mortality rate of influenza H9N2, infectious bronchitis, and Newcastle disease viruses in broiler farms infected to respiratory complex in Iran2015-2016

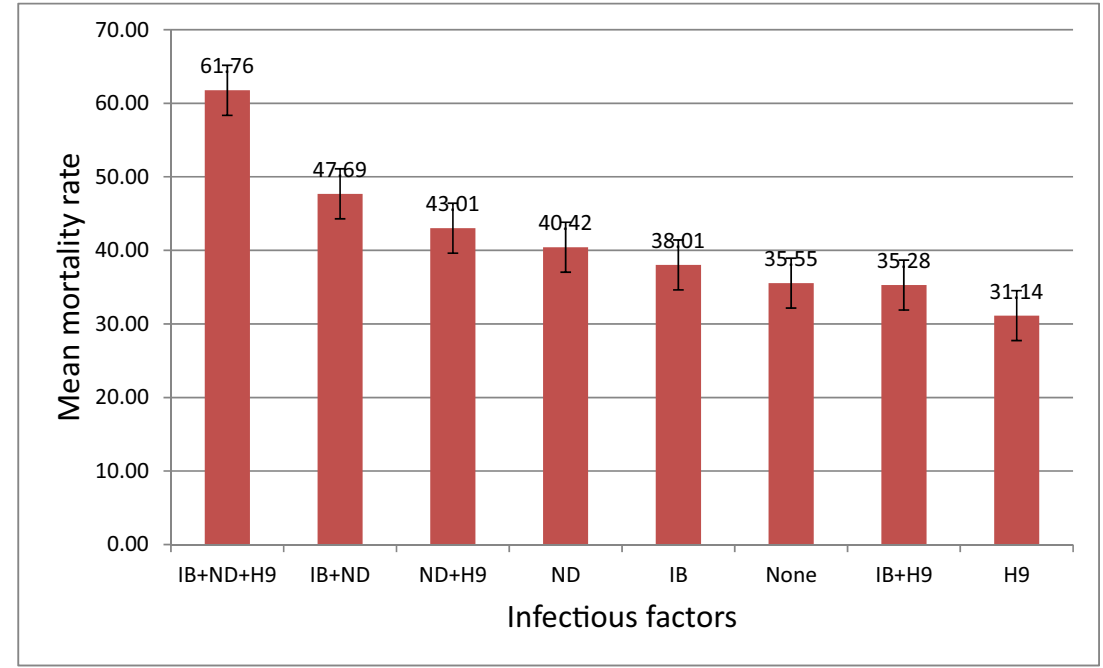


the circulation of variable genotypes of virus in different locations and in diverse time intervals. In addition, there is no vaccination available for establishing cross immunity among disparate exploited genotypes. Recently, a different vaccine of IB, containing H120 and 4/91, has been frequently used, but, notwithstanding this issue, again infectious bronchitis is being counted as a critical complication of broiler farms. Through complete fact-findings over the past years, it seems like the circulating serotypes were in morphism, with serotypes 793/B and variant 2 showing the most capacity and frequency on incidence of the disease (Ghalyanchi-Langeroudi et al. 2015). The continuous observations of circulating viruses and using vaccines similar to these circulating strains, along with observation of health principles and biosecurity issues, are strategies that can be implemented for reduction of disease morbidity to infectious bronchitis.

Apart from infectious bronchitis, Newcastle disease has also had a momentous role in the incidence of respiratory syndromes in the country's broiler farms. Velogenic ND is endemic in Iran and in spite of widespread vaccination, every year, there are outbreaks of Newcastle disease nationwide (Hosseini et al. 2014). In this study, infectious bronchitis, avian influenza, and Newcastle disease were detected in $27 \%$ of the broiler farms surveyed. Recently, distinctive analyses have allowed researchers to understand the active Newcastle disease virus genotype variants in the nation (Hosseini et al. 2014; Mayahi and Esmaelizad 2017; Mehrabanpour et al. 2014; Sabouri et al. 2017). The above-mentioned probes indicate that, despite huge vaccination drives against the disease in broiler farms, we still witness sporadic occurrence of this disease in the country.

Newcastle disease has been a major challenge for poultry breeding farms, not only for Iran but also for other countries as well. In a study executed in broiler farms of Egypt, velogenic ND viruses were diagnosed in $81 \%$ of the studied farms. In Pakistan, numerous velogenic Newcastle genotypes were isolated in different species of birds (Wajid et al. 2017). Furthermore, during the period of 2011-2012, outbreaks of velogenic ND were observed in Israel, Indonesia, and Pakistan (Miller et al. 2015). Molecular epidemiological studies show that these new virus strains from the various geographic regions can replace the dominant strains of individual countries (Miller et al. 2015). Backyard poultry is a reservoir of velogenic Newcastle disease in many countries and it has a critical role in transmitting contamination to commercial farms (A Awan et al. 1994).

Iran also has backyard poultry in many provinces, including the northern provinces where the numbers of industrial poultry and backyard poultry are both high. Since there is a lack of regular and organized vaccination in backyard poultry, the spread of disease from this poultry to industrial poultry is possible.
According to our current exploration, in spite of extensive vaccination in most farms, this disease is one of the most challenging problems in the poultry breeding industry of Iran for diverse reasons including low biosecurity in farms and inadequate vaccination programs in some other farms. The constant monitoring of farms for assessment of virus variations, reviewing of farm vaccination programs, and increasing the biosecurity level are among the essential measures required for control of disease in the country.

Avian influenza H9N2 subtype is also regarded as one of the cogent viral factors in the incidence of respiratory diseases in broiler farms of the nation. The disease is endemic in the country, and apart from this fact that the virus is low pathogenic, co-infection with IBV or NDV results in great economic losses due to severe morbidity and mortality rates in broiler flocks (Nili and Asasi 2002; Pourbakhsh et al. 2008). Avian influenza is endemic not only in Iran but also in neighboring countries. The disease has great economic and public health burdens on national and international trade and travel. In a study done in Egypt, the H9N2 influenza strain was diagnosed in $53 \%$ of samples, and together with infectious bronchitis, the prevalence showed maximum cases of co-infection at $41.7 \%$ (Hassan et al. 2016). In the United Arab Emirates during 2000-2003, the H9N2 influenza virus was isolated from broilers and quails with mortality (Aamir et al. 2007). In Pakistan, this disease has become endemic since 1996, and its annual occurrence has caused the poultry industry there to suffer great economic losses (Cameron et al. 2000; Lee et al. 2016).

In Iran, vaccination against $\mathrm{H} 9 \mathrm{~N} 2$ is being done in breeders and layers as well as in many broiler flocks; however, this disease continues to be destructive.

In the current exploration, the mortality of infectious bronchitis alone was at $38 \%$, and the co-infection of IBV with NDV had risen to $48 \%$, the concomitant infection of IB, $\mathrm{ND}$, and AI hit $67 \%$, but co-infection mortality of IB with H9N2 influenza was at $36 \%$, while it is in contrary with the study of Seifi et al. (2010) and the study of Hassan et al. (2016) that show mortality of co-infection of IB and AIVH9N2 is more than that of IB alone.

\section{Conclusion}

The results of the present study show that the most critical factor in the prevalence of respiratory syndromes in Iran, specifically at the time of doing this groundwork, has been the bronchitis virus with its frequency caused by two serotypes of variant 2 and 793/B. Furthermore, the roles of H9N2 influenza and velogenic Newcastle viruses in the outbreak of respiratory syndromes are distinct, while their simultaneous infection is the cause of virulence in the farms. On the other hand, due to arguments of distinct strains, combined with alternation of 
dominant viruses, the serotypes, genotypes, and circulation of strains between viruses are also exposed to unremitting anamorphic conditions. Thus, necessary measures for strategic control of this process include permanent monitoring of respiratory syndrome cases that have occurred as well as the review of vaccination plans for affected flocks in each year. It is recommended that epidemiologic studies should be conducted frequently on risk factors of prevalence of these diseases and effectiveness of vaccination strategy in the flocks.

Acknowledgements The researchers express their appreciation to the Department of Health and Management of Poultry Diseases of Iranian Veterinary Organization and other related poultry departments of the provinces as well as the national central diagnostic lab of IVO for the implementation of this project.

\section{Compliance with ethical standards}

Conflict of interest The authors declare that they have no conflict of interest.

\section{References}

A Awan, M., J Otte, M., and D James, A., 1994. The epidemiology of Newcastle disease in rural poultry: A review, Avian Pathol. 23, 405423

Aamir, U.B. et al., 2007. Characterization of avian H9N2 influenza viruses from United Arab Emirates 2000 to 2003, Virology, 361, 4555

Aghakhan, S.M. et al., 1994. Studies on Avian Viral Infections in Iran, Arch Razi Inst, 44.45, 1-10

Callison, S.A. et al., 2006. Development and evaluation of a real-time Taqman RT-PCR assay for the detection of infectious bronchitis virus from infected chickens, J Virol Methods, 138, 60-65

Cameron, K.R. et al., 2000. H9N2 subtype influenza A viruses in poultry in pakistan are closely related to the H9N2 viruses responsible for human infection in Hong Kong, Virology, 278, 36-41

Ebadzadeh, H.R. et al., 2015. Agricultural statistics. (Center for Information and Communication Technology, Tehran), 99-160

Ghalyanchi-Langeroudi, A. et al., 2015. Genotyping of Infectious Bronchitis Viruses in the East of Iran, 2015, Iranian J Virol, 9, 31-35

Hadipour, M., and Golchin, P., 2011. Serosurvey of H9N2 avian influenza virus during respiratory disease outbreaks in broiler flocks in Dezful, southern Iran, Bulg J Vet Med, 14, 62-65

Hassan, K.E. et al., 2016. Prevalence of avian respiratory viruses in broiler flocks in Egypt, Poult Sci, 95, 1271-1280

Hosseini, H., Langeroudi, A.G., and Torabi, R., 2014. Molecular characterization and phylogenetic study of Newcastle disease viruses isolated in Iran, 2010-2012, Avian Dis, 58, 373-376
Hosseini, H. et al., 2015. Epidemiology of Avian Infectious Bronchitis Virus Genotypes in Iran (2010-2014), Avian Dis, 59, 431-435

Jackwood, M.W., 2012. Review of infectious bronchitis virus around the world, Avian Dis, 56, 634-641

Kant, A. et al., 1997. Differentiation of virulent and non-virulent strains of Newcastle disease virus within 24 hours by polymerase chain reaction, Avian Pathol, 26, 837-849

Lee, D.-H. et al., 2016. H9N2 low pathogenic avian influenza in Pakistan (2012-2015), Vet Rec Open, 3, e000171

Mayahi, V., and Esmaelizad, M., 2017. Molecular evolution and epidemiological links study of Newcastle disease virus isolates from 1995 to 2016 in Iran, Arch Virol, 162, 3727-3743

Mehrabanpour, M.J. et al., 2014. Phylogenetic characterization of the fusion genes of the Newcastle disease viruses isolated in Fars province poultry farms during 2009-2011, Vet Res Forum: Int Quart J, 5, 187-191

Miller, P.J. et al., 2015. Identification of new sub-genotypes of virulent Newcastle disease virus with potential panzootic features, Infect Genet Evol, 29, 216-229

Modiri Hamadan, A. et al., 2017. Genotyping of Avian infectious bronchitis viruses in Iran (2015-2017) reveals domination of IS-1494 like virus, Virus Res, 240, 101-106

Monne, I. et al., 2008. Development and validation of a one-step real-time PCR assay for simultaneous detection of subtype H5, H7, and H9 avian influenza viruses, J Clin Microbiol, 46, 1769-1773

Naeem, K. et al., 2007. Avian influenza in Pakistan: outbreaks of low- and high-pathogenicity avian influenza in Pakistan during 2003-2006, Avian Dis, 51, 189-193

Nili, H., and Asasi, K., 2002. Natural cases and an experimental study of H9N2 avian influenza in commercial broiler chickens of Iran, Avian Pathol, 31, 247-252

Nili, H., and Asasi, K., 2003. Avian influenza (H9N2) outbreak in Iran, Avian Dis, 47, 828-831

OIE, 2017. Manual of Diagnostic Tests and Vaccines.for Terrestrial Animals 2017. Chapter 2.3.2. Avian infectious bronchitis (NB: Version adopted in May 2013). 2017a,

Pourbakhsh, S.A. et al., 2008. Ninetythree B type, the Predominant Circulating Type of Avian Infectious Bronchitis Viruses 1999 2004 in Iran: a retrospective study, Arch Razi Inst, 63, 1-5

Sabouri, F., Vasfi Marandi, M., and Bashashati, M., 2017. Characterization of a novel VIIl sub-genotype of Newcastle disease virus circulating in Iran, Avian Pathol, 47, 90-99

Seger, W. et al., 2016. Genotyping of infectious bronchitis viruses from broiler farms in Iraq during 2014-2015, Arch Virol, 161, 1229-1237

Seifi, S., Asasi, K., and Mohammadi, A., 2010. Natural co-infection caused by avian inflfl uenza $\mathrm{H} 9$ subtype and infectious bronchitis viruses in broiler chicken farms, Vet Arhiv, 80, 269-281

Swayne, D.E. et al., 2013. Diseases of Poultry, (Blackwell Publishing Ltd., Iowa, USA)

Wajid, A. et al., 2017. Repeated isolation of virulent Newcastle disease viruses in poultry and captive non-poultry avian species in Pakistan from 2011 to 2016, Prev Vet Med, 142, 1-6

Xia, J. et al., 2017. Genetic and antigenic evolution of H9N2 subtype avian influenza virus in domestic chickens in southwestern China, 2013-2016, PLOS ONE, 12, e0171564 